\title{
Vertical transmission and reproduction rate: modeling a common strategy for two related diseases
}

\author{
Abba Mahamane Oumarou ${ }^{1}$ and Yannick Tchaptchie Kouakep ${ }^{2,3^{*}}$ (]
}

\author{
*Correspondence: kouakep@ \\ aims-senegal.org \\ ${ }^{2}$ University of Ngaoundere, \\ P.O. Box 454, Ngaoundere, \\ Cameroon \\ Full list of author information \\ is available at the end of the \\ article
}

\begin{abstract}
Motivated by (Goyal and Murray in PLoS One 9(10):e110143, 2014) we consider a partially age-structured model simulating the dynamic of two infectious diseases vertically transmitted almost independently with horizontal coinfection and a common agestructured vaccination strategy. We study influence of parameters on existence and uniqueness of solutions and epidemiological equilibria. Impact of vertical transmission on basic reproduction rate is also presented.
\end{abstract}

Keywords: Vaccination, Basic reproduction rate, Differential infectivity

Mathematics Subject Classification: 35Q92, 92D30, 92D25

\section{Introduction}

This paper investigates influence of parameters on existence and uniqueness of solutions and equilibria in an age structured model. This model mimics the dynamic of two diseases vertically transmitted almost independently with horizontal coinfection and a common vaccination strategy. For example Goyal and Murray (2014) notes a decrease in Hepatitis B virus ( $\mathrm{HBv}$ ) prevalence as vaccination coverage increases and it is possible to eradicate both $\mathrm{HBv}$ and $\mathrm{HDv}$ (hepatitis $\mathrm{D}$ virus) using high vaccination coverage. Age structure $a$ added to the continuous time $t$ brings some improvements in the comprehension of the disease dynamics. For the case of $\mathrm{HBv}$ and $\mathrm{HDv}$, age plays also a great role in the vaccination strategy (Goyal and Murray 2014). We follow methods of Djidjou et al. (2014), Yang et al. (2014), Brauer et al. (2013) or Inaba (1990) for quantitative (wellposedness with semigroup theory) studies. One can see also Castillo-Chavez and Feng (1998), Greenhalgh (2010) and references Hadeler and Muller (1996), Kouakep and Houpa (2014), Muller (1998, 2000), and Pasquini and Cvjetanović (1988) therein for a good review. Most of time in Africa (WHO 2014), vaccination campaigns concern more than one disease. This study starts with the case of two diseases and forthcoming works will deal with more than two diseases.

We study impact on basic reproduction rate (with a common vaccination strategy) of vertical transmission. Our goal is to bring our contribution with quantitative results concerning special cases in the context of non-linear dynamics of infectious diseases (in the

(c) 2016 Oumarou and Kouakep. This article is distributed under the terms of the Creative Commons Attribution 4.0 International License (http://creativecommons.org/licenses/by/4.0/), which permits unrestricted use, distribution, and reproduction in any medium, provided you give appropriate credit to the original author(s) and the source, provide a link to the Creative Commons license, and indicate if changes were made. 
context of the "Appendix 1") and non local boundary conditions. We included vaccination ignored in Djidjou et al. (2014), vertical transmission neglected in Castillo-Chavez and Feng (1998) and show explicitly the basic reproduction rate theoretical shown with an equivalent number in term of asymptotic properties (threshold parameter) in Djidjou et al. (2014).

The paper is organised as follow: second, third and fourth sections are respectively devoted to problem formulations, primary material on the model, some asymptotic results with impacts on basic reproduction rate induced by vertical transmission. We conclude with a discussion and conclusions.

\section{Problem formulations}

In this work we will consider the following model with vaccination for two diseases $\mathrm{W}$ and Y. $(s, v, I, E, R)$ satisfies (see "Preliminary materials" section for Banach spaces used) the following system of equations:

$$
\begin{aligned}
& \left(\partial_{t}+\partial_{a}+\Psi(a)+\mu\right) s(t, a)=-\lambda(t) s, t, a>0, \\
& \left(\partial_{t}+\partial_{a}+\mu\right) v(t, a)=\Psi(a) s(t, a)-\delta \lambda(t) v(t, a), \\
& I^{\prime}(t)=\lambda(t) \int_{0}^{\infty} p(a)(s(t, a)+\delta v(t, a)) d a-\left(v_{I}+\sigma-\Lambda_{I}\right) I(t), \\
& E^{\prime}(t)=\lambda(t) \int_{0}^{\infty} q(a)(s(t, a)+\delta v(t, a)) d a-\left(v_{E}-\Lambda_{E}\right) E(t)+\sigma I(t), \\
& R^{\prime}(t)=-\mu R(t)+\left(\mu_{I}-\epsilon_{1}\right) I(t)+\left(\mu_{E}-\epsilon_{2}\right) E(t), \quad t>0,
\end{aligned}
$$

posed for time $t>0$, (chronological) age $a>0, \mu_{I}, \mu_{E}, \mu, \epsilon \geq 0, \mu_{E} \geq \mu_{I}$ with recovery rates $\mu_{I}-\epsilon_{1} \geq 0$ and $\mu_{E}-\epsilon_{2} \geq 0$. Here $s(t, a)$ denotes the age-specific density of susceptibles to the two diseases, $E(t)$ and $I(t)$ denote respectively the age-specific densities of infected individuals by diseases $\mathrm{Y}$ and $\mathrm{W}$ (that can be symptomatic or asymptomatic) while $R(t)$ denotes the recovered of both diseases and immunized individuals. $v(t, a)$ is the density of vaccinated individuals for both diseases $W$ and $Y$. We should note that disease $\mathrm{W}$ can make the infection by disease $\mathrm{Y}$ easier: that is why we see the possible transition $\sigma$ from compartment I to compartment $\mathrm{E}$ (see e.g. $\mathrm{W}=$ hepatitis $\mathrm{B}$ and $\mathrm{Y}=$ hepatitis D).

$\Lambda_{I}$ and $\Lambda_{E}$ traduce respectively the proportional influx of new infectives of W- and Y-types coming from vertical transmission (see "Appendix 1"). We will track their influences on basic reproduction rate.

To perform our analysis we shall assume that the contact between individuals is homogeneous so that $\beta_{i}\left(a, a^{\prime}\right) \equiv \beta_{I}>0$ and $\beta_{e}\left(a, a^{\prime}\right) \equiv \beta_{E} \geq 0$ and vaccination strategies are time-independent $\Psi(t, a) \equiv \Psi(a) \geq 0$.

The term $\lambda(t)$ corresponds to the age-specific force of infection and follows the usual law of mass-action, that reads as $\lambda(t)=\beta_{I} I(t)+\beta_{E} E(t)$. Here $\beta_{I}$ and $\beta_{E}$ respectively denote the constant contact transmission rates between $\mathrm{W}$-infected and Y-infected with all the susceptibles. $\Psi(t, a)$ is the proportion of susceptibles with age $a$ vaccinated at time t. $0 \leq \delta \leq 1$ is the reduction in risk due to prior exposure to vaccination (see CastilloChavez and Feng 1998). That means: $\delta=0$ corresponds to a perfect vaccine and $\delta=1$ corresponds to a totally imperfect vaccine. In addition $p \in L_{+}^{\infty}(0, \infty)$ is a given function such that $0 \leq p(a) \leq 1$ a.e. Function $q$ (with $0 \leq q(a) \leq 1$ a.e.) represents the age-specific 
probability to become Y-infected when becoming infected by Y at age $a$. Function $p$ denotes the probability to develop an W-infection when getting the infection $\mathrm{W}$ at age $a$. We refer for the exceptional case of hepatitis B to Edmunds et al. (1993) for more explanation on the age-dependence susceptibility to the infection and their estimations from data with maximum likelihood or least squares methods. This problem is supplemented together with the positive boundary conditions (choosing between $i=1$ and $i=2$ ):

$$
\begin{aligned}
& s(t, 0)=f_{i}\left(\int_{0}^{\omega} \bar{\varepsilon}(a)\left[s(t, a)+l_{1} v(t, a)\right] d a+l_{2} R(t)+l_{3} I(t)+l_{4} E(t)\right) \\
& s(0, a)=s_{0}(a), v(t, 0)=0, v(0, a)=v_{0}(a), I(0)=I_{0}, E(0)=E_{0}, R(0)=R_{0}
\end{aligned}
$$

where $\bar{\varepsilon}(a)=(1-\varepsilon(a))$ and $0 \leq \varepsilon(a) \leq 1$, for a.e $a \geq 0,0 \leq \epsilon_{1} \leq \mu_{I}, 0 \leq \epsilon_{2} \leq \mu_{E}$ and $l_{1}, l_{2}, l_{3}, l_{4} \in[0 ; 1]$. We consider the fertility rate $f$ as constant while defining the functions $f_{1}: x \in \mathbb{R} \mapsto f . x$ and $f_{2}: x \in \mathbb{R} \mapsto \Lambda \in[0 ;+\infty)$. Note that the $R$ component of the system is decoupled from the other components and has therefore no impact upon the long time behaviour of the system. This model is derived from the age structured model presented in "Appendix 1".

Like Republic of Niger's or Cameroonian Governments (see Ministry 2014) we choose the situation with no newborn baby vaccination: $v(t, 0)=0$. Technically in most part of this work, the maximum lifetime $\omega$ will be taken as $+\infty$ (through coefficients' supports) for sake of simplicity.

\section{Preliminary materials}

We point out that the case $i=2$ with $\sigma=0$ has been partially investigated in Kouakep and Houpa (2014) using integrated semigroup theory. We focus later on case $i=1$. In the sequel $\omega \in(0 ;+\infty]$ : it represents biologically the human maximum lifetime.

\section{Abstract formulation}

Let $X$ be the space defined as $X:=\left[L^{1}(0, \omega ; \mathbb{R})\right]^{2} \times \mathbb{R} \times \mathbb{R} \times \mathbb{R}$ endowed with the norm for $\varphi=\left(\varphi_{1}, \varphi_{2}, \varphi_{3}, \varphi_{4}, \varphi_{5}\right)^{T} \in X$ with $\omega$ in $[0,+\infty]$ and

$$
\|\varphi\|_{X}:=\left\|\varphi_{1}\right\|_{L^{1}(0, \omega)}+\left\|\varphi_{2}\right\|_{L^{1}(0, \omega)}+\left|\varphi_{3}\right|+\left|\varphi_{4}\right|+\left|\varphi_{5}\right|
$$

Let $X^{+}$be its positive cone $($of $X): X^{+}:=\left[L_{+}^{1}(0, \omega ; \mathbb{R})\right]^{2} \times \mathbb{R}_{+} \times \mathbb{R}_{+} \times \mathbb{R}_{+}$Let us note $X_{+}$the positive cone of $X$. It is well known that $\left(X,\|\cdot\|_{X}\right)$ is a Banach space (Djidjou et al. 2014). We define the linear operator $A: D(A) \subset X \rightarrow X$ defined by

$$
D(A)=\left\{\varphi \in\left[W^{1,1}(0, \infty)\right]^{2} \times \mathbb{R}^{3} \mid\left(\begin{array}{l}
\varphi_{1}(0) \\
\varphi_{2}(0)
\end{array}\right) \equiv\left(\begin{array}{c}
f^{* *}(\varphi) \\
0
\end{array}\right)\right\}
$$

together with $f^{* *}(\varphi):=f_{i}\left(\int_{0}^{\infty} \bar{\varepsilon}(a)\left[\varphi_{1}(a)+l_{1} \varphi_{2}(a)\right] d a+l_{2} \varphi_{5}+l_{3} \varphi_{3}+l_{4} \varphi_{4}\right)$ and

$$
A\left(\begin{array}{c}
\varphi_{1} \\
\varphi_{2} \\
\varphi_{3} \\
\varphi_{4} \\
\varphi_{5}
\end{array}\right) \equiv\left(\begin{array}{c}
-\varphi_{1}^{\prime}(a)-\mu \varphi_{1}(a) \\
-\varphi_{2}^{\prime}(a)-\mu \varphi_{2}(a) \\
-\left(v_{I}+\sigma-\Lambda_{I}\right) \varphi_{3}(a) \\
-\left(v_{E}-\Lambda_{E}\right) \varphi_{4}(a) \\
-\mu \varphi_{5}(a)
\end{array}\right)
$$


as well as the nonlinear Frechet differentiable and locally Lipschitz map $F: \overline{D(A)} \rightarrow X$ defined by

$$
F\left(\begin{array}{c}
\varphi_{1} \\
\varphi_{2} \\
\varphi_{3} \\
\varphi_{4} \\
\varphi_{5}
\end{array}\right)=\left(\begin{array}{c}
-\left(\beta_{I} \varphi_{3}+\beta_{E} \varphi_{4}+\Psi(a)\right) \varphi_{1}(a) \\
\Psi(a) \varphi_{1}(a)-\delta\left(\beta_{I} \varphi_{3}+\beta_{E} \varphi_{4}\right) \varphi_{2} \\
\left(\beta_{I} \varphi_{3}+\beta_{E} \varphi_{4}\right) \int_{0}^{\infty} p(a)\left(\varphi_{1}(a)+\delta \varphi_{2}(a)\right) d a \\
\left(\beta_{I} \varphi_{3}+\beta_{E} \varphi_{4}\right) \int_{0}^{\infty} q(a)\left(\varphi_{1}(a)+\delta \varphi_{2}(a)\right) d a+\sigma \varphi_{3}(a) \\
\left(\mu_{I}-\epsilon_{1}\right) \varphi_{3}(a)+\left(\mu_{E}-\epsilon_{2}\right) \varphi_{4}(a)
\end{array}\right) .
$$

We set $X_{0}:=\overline{D(A)}$ and $X_{0+}$ the positive cone $\left(X_{0} \cap X^{+}\right)$of $X_{0}$. It is easy (see Djidjou et al. 2014) to see that if $\left(v_{I}+\sigma-\Lambda_{I}\right) \geq \mu$ and $\left(v_{E}-\Lambda_{E}\right) \geq \mu$, then:

1. the operator $A$ satisfy Hille-Yosida property: $(-\mu, \infty) \subset \rho(A)$ with

$$
\left\|(\lambda-A)^{-1}\right\|_{\mathcal{L}(X)} \leq \frac{1}{\lambda+\mu}, \quad \forall \lambda>-\mu .
$$

Moreover we have for each $\lambda>-\mu$ : (1-) $(\lambda-A)^{-1} X^{+} \subset X_{0+}$;

2. $A$ is generator of a $C_{0^{-}}$-semigroup of linear bounded operators;

3. the domain $D(A)$ of operator $A$ is dense in $X_{0}:=\overline{D(A)}$ and $A$ is a closed operator.

Standard methodologies apply to provide (1) and (2) (see Pazy 1983) and (3) is due to the fact that the operator $A$ is generator of a $C_{0}$-semigroup of linear bounded operators (see Corollary 2.5 in Pazy 1983). Therefore, one obtains that System (1)-(2) re-writes as the following densely defined Cauchy problem

$$
\frac{d \phi(t)}{d t}=A \phi(t)+F(\phi(t)), \quad \phi(0)=\left(\varphi_{1}, \varphi_{2}, \varphi_{3}, \varphi_{4}, \varphi_{5}\right)^{T}
$$

\section{Existence and uniqueness of solutions}

For arbitrary $\phi_{0} \in X_{0+}$, we solve (8) as mild solution of the integrated equation (see Djidjou et al. 2014): $\phi(t)=\phi_{0}+A \int_{0}^{t} \phi(s) d s+\int_{0}^{t} F(\phi(s)) d s, \forall t \geq 0$. We obtain the following lemma.

Lemma 1 Assume that $\varepsilon \in L_{+}^{\infty}(0, \omega ; \mathbb{R}), \Lambda_{I} \leq \epsilon_{1}$ and $\Lambda_{E} \leq \epsilon_{2}$, then:

(a) The operator $A$ is generator of a $C_{0}$-semigroup of linear bounded operators and the domain $D(A)$ of operator $A$ is dense in $X_{0}$ and $A$ is a closed operator.

(b) Moreover, the nonlinear operator F from $X$ to $X$ is continuous and locally Lipschitz.

(c) (8) generates a strongly continuous positive semiflow $\{U(t)\}_{t \geq 0}$ on $X_{0+}$. This means that for each $x=\phi(0) \in X_{0+}$, the continuous map $t \rightarrow U(t) x$ defined from $[0, \infty)$ into $X_{0+}$ is a weak solution of (8), that is (for the integrated problem)

$$
\int_{0}^{t} \phi(s) d s \in D(A), \quad \forall t \geq 0, \quad \phi(t)=x+A \int_{0}^{t} \phi(s) d s+\int_{0}^{t} F(\phi(s)) d s, \quad \forall t \geq 0 .
$$

(d) It satisfies the following bounded-dissipative estimates for each $x=\phi(0) \in X_{0+}$ (with $\omega=+\infty$ for sake of simplicity) and each $t \geq 0$ :

$$
\|x\|_{X} e^{-\bar{\Lambda} t} \leq\|U(t) x\|_{X} \leq \frac{\|x\|_{X}}{\underline{\Lambda}}\left(1-e^{-\underline{\Lambda} t}\right)+\|x\|_{X} e^{-\underline{\Lambda} t},
$$


or

$\|x\|_{X} e^{-\bar{\Lambda} t} \leq\|U(t) x\|_{X} \leq \frac{\|x\|_{X}}{\mu}\left(1-e^{-\mu t}\right)+\|x\|_{X} e^{-\mu t}$,

with $\underline{\Lambda}=\mu+\min \left\{\left(\epsilon_{1}-\Lambda_{I}\right),\left(\epsilon_{2}-\Lambda_{E}\right)\right\}$, and $\bar{\Lambda}=\mu+\max \left\{\left(\epsilon_{1}-\Lambda_{I}\right),\left(\epsilon_{2}-\Lambda_{E}\right)\right\}$.

(e) Theorem 1.4 in Pazy (1983) proves that for $\phi_{0} \in X_{0+}=\left[L_{+}^{1}(0, \omega ;[0,+\infty))\right]^{2}$ $\times[0,+\infty)^{3}$ there exists a unique bounded continuous solution $\phi$ to the integrated problem defined on $[0,+\infty)$ with values in $X_{0+}$.

Proof The proof of $[a-b-c-e]$ is rather standard. Indeed it is easy to check that operator $A$ satisfies the Hille-Yosida property. Then standard methodologies apply to provide the existence and uniqueness of mild solution for System (1)-(2) (see for instance Djidjou et al. 2014, Pazy 1983 and the references therein).

We define the total population $P(t)$ at time $t$ by $P(t)=\int_{0}^{+\infty}\left(\phi_{1}(t, a)+\phi_{2}(t, a)\right) d a$ $+\phi_{3}(t)+\phi_{4}(t)+\phi_{5}(t)$ and use the fact that $P(0)=\|x\|_{X}$. The proof of $[d]$ is immediate from the integration of the Eqs. (1)-(2) using formal differentiation of $P(t)$ in respect to $t$ and assumptions made: $\varepsilon \in L_{+}^{\infty}(0, \omega ; \mathbb{R}), \Lambda_{I} \leq \epsilon_{1}$ and $\Lambda_{E} \leq \epsilon_{2}$.

Remark 1 Under assumptions of Lemma 1, one could show that the semiflow $\{U(t)\}_{t \geq 0}$ is asymptotically smooth on $X$ by using results derived by Sell and You (2002).

From Lemma 1 and above Remark 1, one deduces using the results of Hale (1989), Smith and Thieme (2011), and Magal and Zhao (2005) the following results:

Lemma 2 Assume that $\varepsilon \in L_{+}^{\infty}(0, \omega ; \mathbb{R}), \Lambda_{I} \leq \epsilon_{1}$ and $\Lambda_{E} \leq \epsilon_{2}$. The semiflow $\{U(t)\}_{t \geq 0}$ provided by Lemma1 has a non-empty compact global attractor $\mathcal{A} \subset X_{0+}$. It means that $\mathcal{A}$ is compact, invariant and attracts all bounded set $B \subset X_{0+}$, such that for each $B \subset X_{0+}$ bounded subset, one has $d(U(t) B, \mathcal{A}) \rightarrow 0$ as $t \rightarrow \infty$ where $d(B, A)$ denotes the semi distance from $B$ to $A$ defined by $d(B, A)=\sup _{y \in B} \inf _{x \in A}\|y-x\|_{X}$.

\section{Asymptotic properties: impact on basic reproduction rate of vaccination efficiency and vertical transmission}

In all this section we assume that $\varepsilon \in L^{\infty}(0, \omega ; \mathbb{R}), \Lambda_{I}<\epsilon_{1}$ and $\Lambda_{E}<\epsilon_{2}$. We will see that the basic reproduction rate $R_{0}$ is a decrease function of $\Psi$ as noticed by Goyal and Murray (2014).

We denote by $R_{0}^{\Psi}$ the basic reproduction rate with vertical transmission and vaccination for the new model (1)-(2). Then $R_{0}^{\Psi}:=R_{0}^{\Psi}$ (vert) has the value

$$
\left[\int_{0}^{\infty}\left(\frac{\beta_{I} p(a)}{a_{1}}+\frac{\beta_{E} q(a)}{a_{2}}+\frac{\beta_{E} \sigma p(a)}{a_{3}}\right) \times\left(s_{F}(a)+\delta v_{F}(a)\right) d a\right]
$$

where $a_{1}:=\left(v_{I}+\sigma-\Lambda_{I}\right), a_{2}:=\left(v_{E}-\Lambda_{E}\right)$ and $a_{3}:=a_{1} a_{2}$.

\section{Steady states (DFE and EE)}

Here we provide some information on steady states for (1)-(2). 
Lemma 3 Assume that $\forall u \in\{I, E\}, \Lambda_{u}<v_{u}$. The following holds true:

(i) If $R_{0}^{\Psi} \leq 1$, then System (1)-(2)-( $\left.i=1\right)$ has a unique stationary state

$x_{F}=\left(s_{F}(a), v_{F}(a), 0,0,0\right)^{T} \in X_{+}$

where

$$
\begin{aligned}
s_{F}(a)= & f_{i}\left(\int_{0}^{\infty}(1-\varepsilon(u))\left[s_{F}(u)+l_{1} v_{F}(u)\right] d a\right) \\
& \times \exp \left(-\mu a-\int_{0}^{a} \Psi(s) d s\right), \quad a \geq 0,
\end{aligned}
$$

and $v_{F}(a)=\int_{0}^{a} \Psi(s) s_{F}(s) \exp (-\mu(a-s)) d s$.

(ii) If $R_{0}^{\Psi}>1$, then system (1)-(2)-( $\left.i=1\right)$ has two stationary states: Disease free equilibrium (DFE) $x_{F} \in X_{+}$and Endemic Equilibrium $(E E) x_{E}=\left(s_{E}(.), v_{E}(.), I_{E}, E_{E}, R_{E}\right)^{T}$ with

$$
\begin{aligned}
s_{E}(a) & =f_{i}\left(\int_{0}^{\infty} \bar{\varepsilon}(s)\left[s_{E}(s)+l_{1} v_{E}(s)\right] d s\right. \\
& \left.+l_{2} R_{E}+l_{3} I_{E}+l_{4} E_{E}\right) \times \exp \left(-\left(\lambda_{E}+\mu\right) a-\int_{0}^{a} \Psi(s) d s\right), \\
v_{E}(a) & =\int_{0}^{a} \Psi(s) s_{E}(s) \exp \left(-\left(\delta \lambda_{E}+\mu\right)(a-s)\right) d s, \\
I_{E} & =\frac{\lambda_{E}}{v_{I}+\sigma-\Lambda_{I}} \int_{0}^{\infty} p(a)\left(s_{E}(a)+\delta v_{E}(a)\right) d a, \\
E_{E} & =\frac{\lambda_{E}}{v_{E}-\Lambda_{E}} \int_{0}^{\infty} q(a)\left(s_{E}(a)+\delta v_{E}(a)\right) d a+\frac{\sigma}{v_{E}-\Lambda_{E}} I_{E} \\
R_{E} & =\frac{\left(\mu_{I}-\epsilon_{1}\right)}{\mu} I_{E}+\frac{\left(\mu_{E}-\epsilon_{2}\right)}{\mu} E_{E} .
\end{aligned}
$$

where $\lambda_{E}>0$ is the unique solution of the equation

$$
\begin{aligned}
1= & \int_{0}^{\infty}\left(\frac{\beta_{I}}{v_{I}+\sigma-\Lambda_{I}} p(a)+\frac{\beta_{E}}{v_{E}-\Lambda_{E}} q(a)\right. \\
& \left.+\frac{\beta_{E} \sigma p(a)}{\left(v_{E}-\Lambda_{E}\right)\left(v_{I}+\sigma-\Lambda_{I}\right)}\right)\left(s_{E}(a)+\delta v_{E}(a)\right) d a
\end{aligned}
$$

Rewriting Eq. (13) provides (see also Inaba 2001) a coupled integral equations system. The existence and uniqueness of continuous solutions $\left(s_{E}, v_{E}, I_{E}, E_{E}, R_{E}\right)$ for this type of Volterra like system is given by Gurtin and MacCamy (1974) (see a special case in "DFE special case $\left(s^{*}, v^{*}, I^{*}=0, E^{*}=0, R^{*}=0\right)$ : integral equation" and "EE special case ( $s^{*}, v^{*}$, $\left.I^{*} \neq E^{*} \neq 0, R^{*} \neq 0\right)$ : integral equation" sections in "Appendix 2".

\section{Threshold number explained as basic reproduction rate}

We recall $R_{0}^{\Psi}$ the basic reproduction rate with vertical transmission and vaccination for the model (1)-(2) (see "Appendix 1"). Clearly it is denoted $R_{0}^{\Psi}:=R_{0}^{\Psi}$ (vert) with the value

$$
\left[\int_{0}^{\infty}\left(\frac{\beta_{I} p(a)}{a_{1}}+\frac{\beta_{E} q(a)}{a_{2}}+\frac{\beta_{E} \sigma p(a)}{a_{3}}\right) \times\left(s_{F}(a)+\delta v_{F}(a)\right) d a\right]
$$


where $a_{1}:=\left(v_{I}+\sigma-\Lambda_{I}\right)$, and $a_{2}:=\left(v_{E}-\Lambda_{E}\right)$ with $a_{3}:=a_{1} a_{2}$. We recall the basic reproduction rate for our model with vertical transmission without vaccination

$$
R_{0}^{0}(\text { vert }):=\left[\int_{0}^{\infty}\left(\frac{\beta_{I}}{a_{1}} p(a)+\frac{\beta_{E}}{a_{2}} q(a)+\frac{\beta_{E} \sigma}{a_{3}} p(a)\right) s_{F}(a) d a\right]
$$

and the basic reproduction rate for our model without vaccination and transition $\sigma=0$ nor vertical transmission

$$
R_{0}^{0}(\text { novert }):=\left[\int_{0}^{\infty}\left(\frac{\beta_{I}}{v_{I}} p(a)+\frac{\beta_{E}}{v_{E}} q(a)\right) s_{F}(a) d a\right]
$$

or the basic reproduction rate $R_{0}^{\Psi}$ (novert) for our model with vaccination but vertical transmission and transition $\sigma=0$ excluded [see also Kouakep and Houpa (2014) with $\Lambda_{E}=0, \Lambda_{I}=0$ and $\left.i=2\right]$ is given by

$$
\left[\int_{0}^{\infty}\left(\frac{\beta_{I}}{v_{I}} p(a)+\frac{\beta_{E}}{v_{E}} q(a)\right)\left(s_{F}(a)+\delta v_{F}(a)\right) d a\right]
$$

Remark 2 It is obvious that the vertical transmission increases the basic reproduction rate if $\forall u \in\{I, E\}, \Lambda_{u}<\epsilon_{d(u)} \leq \mu_{u} \leq v_{u}$ [more deaths than births, $d(I)=1$ and $d(E)=2$ ]. We focus on the case $(i=1)$.

Theorem 1 Assume that $\left(v_{E} \leq \Lambda_{E}\right.$ or $\left.v_{I}+\sigma \leq \Lambda_{I}\right)$ and $h:=\mu+v_{I}+v_{E}-\left(\Lambda_{I}+\Lambda_{E}\right)$ $>0$. Then System (1)-(2)-( $i=1)$ has a unique stationary state (see integral equations in Gurtin and MacCamy 1974; Krasnov et al. 1977) $x_{F}=\left(s_{F}(a), v_{F}(a), 0,0,0\right)^{T} \in X_{+}$where

$$
s_{F}(a)=f_{i}\left(\int_{0}^{\infty} \bar{\varepsilon}(u)\left[s_{F}(u)+l_{1} v_{F}(u)\right] d u\right) \times e^{-\left(\mu a+\int_{0}^{a} \Psi(s) d s\right)}
$$

$a \geq 0$ and $v_{F}(a)=\int_{0}^{a} \Psi(s) s_{F}(s) \exp (-\mu(a-s)) d s$. Moreover, under its assumptions, results in Lemma 1 and Remark 1 on asymptotically smoothness of semiflow hold with this modification:

$$
\|x\|_{X} e^{-\bar{\Lambda} t} \leq\|U(t) x\|_{X} \leq \frac{\|x\|_{X}}{h}\left(1-e^{-h t}\right)+\|x\|_{X} e^{-h t},
$$

Remark 3 Authors like El-Doma (2006) choose to rewrite solution of the PDE (1)(2) $(i=1)$ along characteristics and then construct a Lipschitz operator whose unique global in time fixed point will be the solution in Hadamard sense of the PDE.

\section{Discussion}

The works of Castillo-Chavez and Feng (1998) and Djidjou et al. (2014) are more general by considering age-dependent death rates and birth fertility. But, our work connects these two important works in some of their complementary lacks and strength in order to study the impact on basic reproduction rate (with influence vertical transmission) of a common vaccination strategy inducing the stability of steady states of two related diseases. We saw that the basic reproduction rate $R_{0}$ is a decrease function of $\Psi$ confirming the decrease in Hepatitis $B$ virus $(\mathrm{HBv})$ prevalence as vaccination coverage 
increases (Goyal and Murray 2014): it is then possible to eradicate both $\mathrm{HBv}$ and $\mathrm{HDv}$ (hepatitis D virus) using high vaccination coverage. In further work we will include migrations in the infected individuals' classes. One could biologically suspect the cases $\left(v_{u} \leq \Lambda_{u}, \forall u \in\{E, I\}\right)$ in Lemma 1 to be critical since we would like to avoid blow-up of solutions in order to obtain global in time solutions. We said nothing in the cases: $v_{E}>\Lambda_{E}>\epsilon_{2}$ or $v_{I}+\sigma>\Lambda_{I}>\epsilon_{1}$ What arises in Theorem 1 if $h=0$ ?

\section{Conclusions}

The main objective of this work is study the impact of vertical transmission on basic reproduction rates in the case of coinfection like HBV(hepatitis B)/HDV(hepatitis D) co-infection. We found that vertical transmission increases the basic reproduction rate. Beside this, we studied the influence of the influx by migration on the wellposedness of the mathematical problem: there is a trade-off between entries balanced by mortalities and wellposedness for long term dynamic of our age-structured model. Some asymptotic relations between the mean of the fertility rate and other biological parameters are derived in endemic or free epidemic situations ("Appendices 1 and 2"). A perspective could be to introduce diffusion in our model and evaluate a minimal speed for travelling wave solutions.

\section{Authors' contributions}

$\mathrm{AMO}$ contributes for the modeling of the diseases dynamics, computation of steady states and the discussion around impacts of vertical transmission on basic reproduction rate. While YTK contributes in the model construction, basic reproduction rate design and the proof of the well-posedness of the model with developments in the "Appendices 1 and 2". Both the authors read and approved the final manuscript.

\section{Author details}

${ }^{1}$ Department of Mathematics, University Abdou Moumouni, P.O. Box 10 662, Niamey, Niger. ${ }^{2}$ University of Ngaoundere, P.O. Box 454, Ngaoundere, Cameroon. ${ }^{3}$ Department of Mathematics and Computer Science, AIMS - Cameroon, P.O. Box 608, Limbe, Cameroon.

\section{Acknowledgements}

We would like to thank the two anonymous reviewers and our advisors (Prof B. Mampassi, Prof B. Saley, Prof Békollè D., Prof Houpa D. D. E.), Dr A. Tall (AIMS-Sn), Dr Ntyam A., LYCLAMO/MINESEC with GDM-MIAP student group for their valuable comments or advices. YTK was supported with an AMMSI scholarship through an IMU-CDC Grant in 2014. Research supported also for YTK by Canada's International Development Research Centre (IDRC), and within the framework of the AIMS Research for Africa Project, while AMO was supported by a Post AIMS-Senegal Grant in 2014.

\section{Competing interests}

Both authors declare no competing interest.

\section{Appendix 1: Age structured model}

Our model is derived (through $I(t):=\int_{0}^{\infty} i(t, a) d a, E(t):=\int_{0}^{\infty} e(t, a) d a$ and $\left.R(t):=\int_{0}^{\infty} r(t, a) d a\right)$ from:

$$
\begin{aligned}
\left(\partial_{t}+\partial_{a}+\Psi(t, a)+\mu\right) s(t, a) & =-\lambda_{0}(t, a) s(t, a), \\
\left(\partial_{t}+\partial_{a}+\mu\right) v(t, a) & =\Psi(t, a) s(t, a)-\delta \lambda_{0}(t, a) v(t, a), \\
\left(\partial_{t}+\partial_{a}+\left(\mu_{I}+\mu\right)\right) i(t, a) & =\lambda_{0}(t, a) p(a) \times(s(t, a)+\delta v(t, a))-\sigma i(t, a), \\
\left(\partial_{t}+\partial_{a}+\mu_{E}+\mu\right) e(t, a) & =\lambda_{0}(t, a) q(a) \times(s(t, a)+\delta v(t, a))+\sigma i(t, a), \\
\left(\partial_{t}+\partial_{a}+\mu\right) r(t, a) & =\left(\mu_{I}-\epsilon_{1}\right) i(t, a)+\left(\mu_{E}-\epsilon_{2}\right) e(t, a),
\end{aligned}
$$

posed for time $t>0$, (chronological) age $a>0, \mu_{I}, \mu_{E}, \mu, \epsilon \geq 0, \mu_{E} \geq \mu_{I}$ with recovery rates $\mu_{I}-\epsilon_{1} \geq 0$ and $\mu_{E}-\epsilon_{2} \geq 0$. Here $s(t, a)$ denotes the age-specific density of susceptibles to the two diseases, $e(t, a)$ and $i(t, a)$ denote respectively the age-specific 
densities of infected individuals by diseases $\mathrm{W}$ and $\mathrm{Y}$. The term $\lambda_{0}(t, a)$ corresponds to the age-specific force of infection reads as

$$
\lambda_{0}(t, a)=\int_{0}^{\infty}\left[\beta_{i}\left(a, a^{\prime}\right) i\left(t, a^{\prime}\right)+\beta_{e}\left(a, a^{\prime}\right) e\left(t, a^{\prime}\right)\right] d a^{\prime} .
$$

$v_{I}:=\mu_{I}+\mu$ and $\nu_{E}:=\mu_{E}+\mu$ and considering the fertility rate $f$ as constant while defining the functions $f_{1}: x \in \mathbb{R} \mapsto f . x$ and $f_{2}: x \in \mathbb{R} \mapsto \Lambda \in[0 ;+\infty)$ with $w_{k, c}(t, a)=k . i(t, a)+c . e(t, a)$ and

$$
\begin{aligned}
s(t, 0) & =\int_{0}^{\infty} f(a) b(a)\left(\bar{\varepsilon}(t, a)\left[s(t, a)+l_{1} r(t, a)\right]+w_{1,1}(t, a)\right) d a, \\
i(t, 0) & =\Lambda_{I} \int_{0}^{\infty} i\left(t, a^{\prime}\right) d a^{\prime}: w_{k, c} \equiv w_{1,0} \\
e(t, 0) & =\Lambda_{E} \int_{0}^{\infty} e\left(t, a^{\prime}\right) d a^{\prime}: w_{k, c} \equiv w_{0,1} \\
r(t, 0) & =0, \text { (no immunity at birth), }
\end{aligned}
$$

The "sanitary" coefficient $\varepsilon(a) \in[0 ; 1]$ describes the facility for an individual of age $a$ to transmit vertically disease. If $\varepsilon=0$ the person can not transmit vertically the disease (he/she is "clean"). If $\varepsilon=1$ the person surely transmits vertically the disease (he/she is "totally infective"). $\bar{\varepsilon}(a)=(1-\varepsilon(a))$.

$f(a)$ is the fertility rates while $b \in L_{+}^{\infty}(0, \infty)$ is a given function such that $0 \leq b(a) \leq 1$, for a.e $a \geq 0$ and initial positive data $s(0, a)=s_{0}(a)$ and

$$
i(0, a)=i_{0}(a), \quad e(0, a)=e_{0}(a), \quad r(0, a)=r_{0}(a), \quad v(0, a)=v_{0}(a) .
$$

$b(a)$ is the specific probability to born susceptible.

\section{Appendix 2: Averaged fertility rate}

\section{DFE special case $\left(s^{*}, v^{*}, I^{*}=0, E^{*}=0, R^{*}=0\right)$ : integral equation}

Straightforward computations lead for $a>0$ to:

$$
\begin{aligned}
& s^{*}(a)=f_{i}\left(\int_{0}^{\infty} \bar{\varepsilon}(u)\left[s^{*}(u)+l_{1} v^{*}(u)\right] d u\right) \exp \left(-\mu a-\int_{0}^{a} \Psi(s) d s\right), \\
& s^{*}(0)=f_{i}\left(\int_{0}^{\infty} \bar{\varepsilon}(u)\left[s^{*}(u)+l_{1} v^{*}(u)\right] d u\right), \\
& v^{*}(a)=\int_{0}^{a} \Psi(s) s^{*}(s) \exp (-\mu(a-s)) d s \\
& v^{*}(0)=0, \quad E^{*}=I^{*}=R^{*}=0 .
\end{aligned}
$$

By setting $i=1, \varepsilon(u)=1-\alpha \exp (-\gamma u), \forall u \geq 0$ and $l_{1}=1$ with $w^{*}:=s^{*}+v^{*}$, one obtains:

$$
\begin{aligned}
& w^{*}(a)=\int_{0}^{\infty} w^{*}(u) f(u)(1-\varepsilon(u)) d u \times \exp (-\mu a), \quad a>0, \\
& w^{*}(0)=s^{*}(0) .
\end{aligned}
$$




$$
k(a, u)=(1-\varepsilon(u)) \exp (-\mu a)
$$

with some regularity on $\varepsilon$ ) or with $f(a) \equiv f \geq 0$

$$
w^{*}(a)=f \int_{0}^{\infty} k(a, u) w^{*}(u) d u, a>0, \quad w^{*}(0)=s^{*}(0) .
$$

This is a second type homogeneous Fredholm equation. We have: $\int_{0}^{\infty} \int_{0}^{\infty} k(a, u) d a d u$ finite. Krasnov et al. (1977) justify existence of solutions $w^{*}$ with associated characteristics value(s) $f$. By setting again (in the special case $f(u) \equiv f \geq 0$, $\varepsilon(u)=1-\alpha \exp (-\gamma u)) \geq 0$, and $k(a, u)=\alpha \exp (-(\gamma u+\mu a))$ with $0 \leq \gamma, \alpha \leq 1$ we get

$$
w^{*}(a)=f \int_{0}^{\infty} k(a, u) w^{*}(u) d u, \quad a>0,
$$

In fact, we could biologically and mathematically replace $\int_{0}^{\infty} \int_{0}^{\infty}$ by $\int_{0}^{\omega} \int_{0}^{\omega}$ with $\omega$ the maximal human admissible lifetime (e.g. $\omega=140$ years). Rewriting Eq. (24) along characteristics provides (see also Inaba 2001) a coupled integral equations system. The existence and uniqueness of continuous solutions $\left(s^{*}, v^{*}\right)$ for this type of Volterra like system is also given by Gurtin and MacCamy (1974).

Let consider the problem

$$
w^{*}(a)=f \int_{0}^{\omega} k(a, u) w^{*}(u) d u, \quad a>0
$$

where $f$ is a characteristic value. We found $f=\left(\frac{\gamma+\mu}{\left(1-e^{-(\gamma+\mu) \omega}\right) \alpha}\right)$ associated to the eigenfunction $m^{*}(a)=\exp (-\mu a)$. If $\omega \rightarrow \infty$, then $f \rightarrow\left(\frac{\gamma+\mu}{\alpha}\right)$.

EE special case $\left(s^{*}, v^{*}, I^{*} \neq 0, E^{*} \neq 0, R^{*} \neq 0\right)$ : integral equation

Straightforward computations lead for $a>0$ to:

$$
\begin{aligned}
& s^{*}(a)=f_{i}\left(\int_{0}^{\infty} \bar{\varepsilon}(u)\left[s^{*}(u)+l_{1} v^{*}(u)\right] d u\right) \times \exp \left(-\mu a-\int_{0}^{a} \Psi(s) d s\right), \\
& s^{*}(0)=f_{i}\left(\int_{0}^{\infty} \bar{\varepsilon}(u)\left[s^{*}(u)+l_{1} v^{*}(u)\right] d u\right), \\
& v^{*}(a)=\int_{0}^{a} \Psi(s) s^{*}(s) \exp (-\mu(a-s)) d s \\
& v^{*}(0)=0, \quad E^{*}, I^{*}, R^{*}>0 .
\end{aligned}
$$

By setting $i=1, \delta \approx 1, \varepsilon(u)=1-\alpha \exp (-\gamma u), \forall u \geq 0$ and $l_{1}=1$ with $w^{*}:=s^{*}+v^{*}$, one obtains:

$$
\begin{aligned}
& w^{*}(a)=\int_{0}^{\infty} w^{*}(u) f(u)(1-\varepsilon(u)) d u \times \exp \left(-\left(\mu+\lambda_{E}\right) a\right), \quad a>0, \\
& w^{*}(0)=s^{*}(0) .
\end{aligned}
$$

by setting a degenerated kernel (see Krasnov et al. 1977, p. 59-72) as

$$
k_{1}(a, u)=(1-\varepsilon(u)) \exp \left(-\left(\mu+\lambda_{E}\right) a\right)
$$

with some regularity on $\varepsilon$ ) or with $f(a) \equiv f \geq 0$ 


$$
w^{*}(a)=f \int_{0}^{\infty} k_{1}(a, u) w^{*}(u) d u, \quad a>0, \quad w^{*}(0)=s^{*}(0)
$$

Krasnov et al. (1977) justify existence of solutions $w^{*}$ with associated characteristic value (s) $f$.

By setting again (in the special case $f(u) \equiv f \geq 0, \varepsilon(u)=1-\alpha \exp (-\gamma u)$ ), and $k_{1}(a, u)=\alpha \exp \left(-\left(\gamma u+\left(\mu+\lambda_{E}\right) a\right)\right)$ with $0 \leq \gamma \leq 1$ we get

$$
w^{*}(a)=f \int_{0}^{\infty} k_{1}(a, u) w^{*}(u) d u, \quad a>0,
$$

\section{Let consider the problem}

$$
w^{*}(a)=f \int_{0}^{\omega} k_{1}(a, u) w^{*}(u) d u, \quad a>0
$$

where $f$ is a characteristic value. We found $f=\left(\frac{\gamma+\mu+\lambda_{E}}{\left(1-e^{-\left(\gamma+\mu+\lambda_{E}\right) \omega}\right) \alpha}\right)$ associated to the eigenfunction $m^{*}(a)=\exp \left(-\left(\mu+\lambda_{E}\right) a\right)$. If $\omega \rightarrow \infty$, then $f \rightarrow\left(\frac{\gamma+\mu+\lambda_{E}}{\alpha}\right)$.

Received: 20 November 2015 Accepted: 4 April 2016

Published online: 14 April 2016

\section{References}

Brauer F, Shuai Z, van den Driessche P (2013) Dynamics of an age-of-infection cholera model. Math Biosci Eng 10(5 \& 6):1335-1349

Castillo-Chavez C, Feng Z (1998) Global stability of an age-structure model for TB and its applications to optimal vaccination strategies. Math Biosci 151:135-154

Djidjou DR, Tewa JJ, Bowong S (2014) Analysis of an age-structured SIL model with demographics process and vertical transmission. Special issue CARI'12 in ARIMA J 17:23-52

Edmunds WJ, Medley GF, Nokes DJ, Hall AJ, Whittle HC (1993) The influence of age on the development of the hepatitis B carrier state. Proc R Soc Lond B Biol Sci 253:197-201

El-Doma M (2006) Analysis of an SIRS age-structured epidemic model with vaccination and vertical transmission of disease. AAM 1(1):36-61. http://pvamu.edu/pages/398/asp

Goyal A, Murray JM (2014) The Impact of vaccination and antiviral therapy on hepatitis B and hepatitis D epidemiology. PLoS One 9(10):e110143

Greenhalgh D (2010) Age-structured models and optimal control in mathematical epidemiology: a survey. In: Optimal control of age-structured populations in economy, demography and the environment. Routledge Explorations in Environmental Economics. Routledge, Taylor \& Francis Group, pp 174-206

Gurtin ME, MacCamy RC (1974) Non-linear age-dependant population dynamics. Arch Ration Mech Anal 54:281-300

Hale JK (1989) Asymptotic behavior of dissipative systems. AMS, Providence

Hadeler KP, Muller J (1996) Vaccination in age structured populations II: optimal vaccination strategies. In: Isham V Medley G (eds) Models for infectious human diseases: their structure and relation to data. Cambridge University, Cambridge, pp 102-114

Inaba H (1990) Threshold and stability results for an age-structured epidemic model. J Math Biol 28:411-434

Inaba H (2001) Kermack and MacKendrick revisited: the variable susceptibility model for infectious diseases, Japan. J Ind Appl Math 18:273-292

Krasnov M, Kisselev A, Makarenko G (1977) Équations intégrales: problèmes et exercices. MIR, French trad

Kouakep TY, Houpa DDE (2014) Optimal test strategies for hepatitis B vaccination with no vertical transmission. Gen Math Notes 20(1):19-26

Magal P, McCluskey CC, Webb GF (2010) Liapunov functional and global asymptotic stability for an infection-age model. Appl Anal 89:1109-1140

Magal P, Zhao X-Q (2005) Global attractors and steady states for uniformly persistent dynamical systems. SIAM J Math Anal 37:251-275

Ministry of Public Health of Cameroon, Plan D'introduction du vaccin contre l'Hépatite virale B dans le PEV de routine 2005-2009. http://www.gavialliance.org/country/cameroon/documents/proposals/vaccine-introduction-plan-fornvs---hep-b-support--cameroon--fran\%C3\%A7ais-/. Accessed Feb 2014

Muller J (1998) Optimal vaccination patterns in age-structured populations. SIAM J Appl Math 59(1):222-241 
Muller J (2000) Optimal vaccination patterns in age-structured populations: endemic case. Math Comput Model 31:149-160

Pasquini P, Cvjetanović B (1988) Mathematical models of hepatitis B infection. Ann Ist Super Sanitá 24(2):245-250 Pazy A (1983) Semigroups of linear operators and applications to partial differential equations. Springer, Berlin Sell GR, You Y (2002) Dynamics of evolutionary equations. Springer, New York Smith HL, Thieme HR (2011) Dynamical systems and population persistence. AMS, Providence WHO, Media Center. http://www.who.int/mediacentre/factsheets/. Accessed 01 Aug 2014

Yang J, Qiu Z, Li X (2014) Global stability of an age-structured cholera model. Math Biosci Eng 11(3):641-665

Submit your manuscript to a SpringerOpen ${ }^{\circ}$ journal and benefit from:

- Convenient online submission

- Rigorous peer review

- Immediate publication on acceptance

- Open access: articles freely available online

- High visibility within the field

- Retaining the copyright to your article 